\title{
20. On the Class-number of the Maximal Real Subfield of a Cyclotomic Field
}

\author{
By Hiroyuki OSADA \\ Department of Mathematics, National Defense Academy \\ (Communicated by Shokichi IYANAGA, M. J. A. , April 12, 1993)
}

Let $p$ be a prime. $h^{+}(p)$ will denote as usual the class-number of the maximal real subfield $\boldsymbol{Q}\left(\zeta_{p}+\zeta_{p}^{-1}\right)$ of the cyclotomic field $\boldsymbol{Q}\left(\zeta_{p}\right), \zeta_{p}=e^{\frac{2 \pi i}{p}}$. Under the generalized Riemann Hypothesis $h^{+}(163)$ can be proved to be 4 , but all values of $h^{+}(p)$ hitherto determined are 1 (see [4]). In a series of papers [3], we have obtained some results on $h^{+}(p)$ under the assumption:

(H) $\quad h^{+}(p)<p$.

In particular, we have shown under $(\mathrm{H})$ that

$$
h^{+}(1229)=h^{+}(4493)=3
$$

and

$$
h^{+}(607)=h^{+}(1894)=4 \text {, }
$$

so that, in any case, $h^{+}(p)>1$ for $p=1229,4493,607$ or 1879 . We recall furthermore that the results of [3] were derived from the following proposition:

Proposition. Let $p$ and $q$ be distinct primes. Let $F$ be a finite algebraic number field. Suppose $E / F$ is a Galois $q$-extension and $f$ is the order of $p \bmod q$. Then for any $\alpha$ with $0 \leq \alpha<f$.

(See [3]).

$$
p^{\alpha}\left\|h(E) \Rightarrow p^{\alpha}\right\| h(F) \text {. }
$$

Here and in what follows, $h(L)$ means the class-number of the algebraic number field $L$.

We shall prove in this note, which will be the last paper of this series, that the following theorem follows also from the above proposition:

Theorem. Let $q$ be an odd prime such that $p=8 q+1$ is also a prime. We assume the following condition:

(C) $q+1$ is not a power of $2,2 q+1$ is not a power of $3,4 q+1$ is not a power of 5 and $7 q+1$ is not a power of 2 . Then

$$
h^{+}(p)<p \text { and } h(k(p)) \geqq 5 \Rightarrow h^{+}(p)=h(k(p))
$$

where $k(p)$ is the unique quartic subfield of $\boldsymbol{Q}\left(\zeta_{p}\right)$ over $\boldsymbol{Q}$.

Proof. Since $8 \cdot 3+1=25$, we may assume $q \geqq 5$. Put $K=\boldsymbol{Q}\left(\zeta_{p}+\right.$ $\zeta_{p}^{-1}$ ) and $k=k(p)$. Then $K / k$ is a $q$-extension and the above proposition can be applied.

If $q \nmid h(k)$, then $q \nmid h(K)$ (see [2]). Since $h(K)<p, h(k)<p$. It is easy to show that if $q \mid h(k)$, then $q \| h(k)$ and $q \| h(K)$. Now let $r$ be an odd prime. If $r \equiv 1(\bmod q), r \mid h(k)$ and $r \mid h(K)$, then $r=1+2 n q$, where $n=1$ or 2 or 3 . Since $r^{2}>p$, we have that $r\|h(k), r\| h(K)$. If $r \equiv 1(\bmod q)$ and $r \nmid h(k), r \mid h(K)$, then $h(K) \geqq r \cdot h(k) \geqq 5 r>p$. Hence we have that 
$r \times h(k) \Rightarrow r \mid h(K)$. Now $f>1$ is the order of $r \bmod q$. We will show that $r^{f}>p$.

In case $r \geqq 11, r^{f}-1=(r-1)\left(r^{f-1}+\cdots+1\right)$ can not be $2 n q$, where $n=1$ or 2 or 3 .

Let $r=7$ and $7^{f}=1+2 n q$, where $n=1$ or 2 or 3 . Then $f$ is even. Now let $f=2 m$ for some integer $m$. Hence $\left(7^{m}-1\right)\left(7^{m}+1\right)=2 n q$, where $n=1$ or 2 or 3 . This is a contradiction.

Let $r=5$ and $5^{f}=1+2 n q$, where $n=1$ or 3 .

Let $5^{f}=1+2 q$. Then $f$ is even. Now let $f=2 m$ for some integer $m$. Hence $\left(5^{m}-1\right)\left(5^{m}+1\right)=2 q$. This is a contradiciton.

Let $r=5$ and $5^{f}=1+6 q$. Then $f$ is even. Now let $f=2 m$ for some integer $m$. Hence $\left(5^{m}-1\right)\left(5^{m}+1\right)=6 q$. This is a contradiction.

Let $r=3$ and $3^{f}=1+2 n q$, where $n=2$ or 3 . Then $f$ is even. Now let $f=2 m$ for some integer $m$. Hence $\left(3^{m}-1\right)\left(3^{m}+1\right)=2 n q$, where $n=2$ or 3 . This is a contradiction.

Next $r=2$ and $2^{f}=1+3 q$ or $2^{f}=1+5 q$ or $2^{f}=1+7 q$, then we have that $f=2 m$ for some integer $m$. Since $\left(2^{m}-1\right)\left(2^{m}+1\right)=3 q$, we should have $m=2, q=5$. Therefore we have $2^{f} \neq 1+3 q$. If $2^{f}=1+5 q$, then we have $f=4 m$ for some integer $m .2^{f}-1=\left(4^{m}-1\right)\left(4^{m}+1\right)=5 q$ and $3 \mid 4^{m}-1$, we have that $2^{f} \neq 1+5 q$.

Examples. Suppose $p=857$ or 2153. Suppose $h^{+}(p)<p$. Then $h^{+}(p)=h(k(p))=5$ (see [1]).

Remark. Let $q$ and $p=8 q+1$ be primes. Then we have only 5 examples $\{3,7,13,127,1093\}$ for $q<10^{8}$, which do not satisfy the condition (C) in the theorem.

\section{References}

[1] M. N. Gras: Table Numérique du Nombre de Classes et des Unités des Extensions Cycliques de Degré 4 de $\boldsymbol{Q}$. Publ. math. fasc., 2, Fac. Sci. Besancon (1977/1978).

[2] J. Masely: Class numbers of real cyclic field with small conductors. Compositio Math., 37, 297-319 (1978).

[ 3 ] H. Osada: A remark on the class-number of the maximal real subfield of a cyclotomic field. Proc. Japan Acad., 65A, 318-319 (1989); ditto. II, III. ibid., 68A, $41-42 ; \quad 237-238$ (1992).

[ 4 ] L. C. Washington: Introduction to Cyclotomic Field. Springer (1982). 